\section{Vertebrate face in profile}

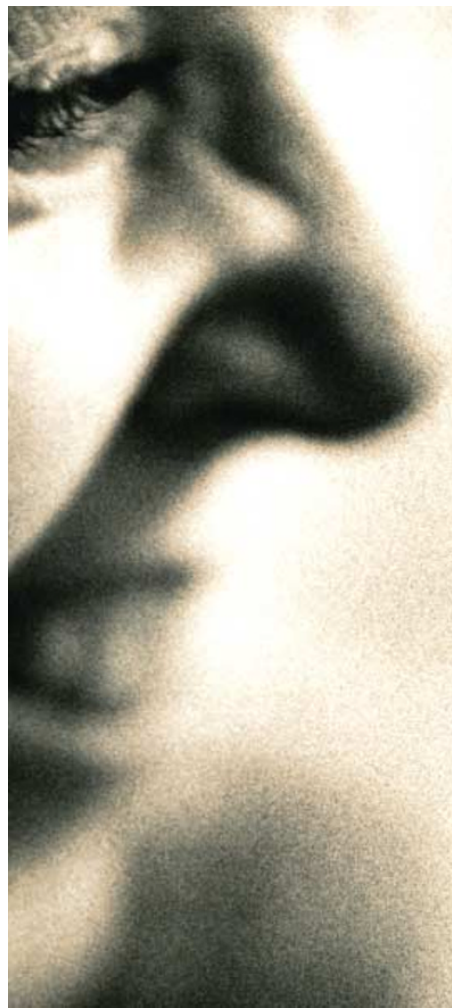

Faces are one of the most distinctive parts of our anatomy; we use them to tell people apart and to express our opinions and emotions. But how do skeletal, muscle and nerve tissues combine to produce this most engaging part of our body? By analysing a zebrafish mutant, Justin Gage Crump and colleagues show that precise hierarchical interactions between the three tissue types coordinate their assembly in one particular segment of the face.

Vertebrate faces come in many guises but they all develop from seven segmented regions (the pharyngeal arches), among which are intercalated outpockets of endodermal tissue, called 'pouches'. Cranial neural crest cells populate the arches and give rise to cartilage, which the pouches support and pattern. Several mutations disrupt the delicate process that controls the morphogenesis and differentiation of skeletal precursors. For example, mutations in the genes that encode integrins have shown that this conserved family of heterodimeric receptors are involved in signalling and are required for the migration of neural crest cells to the arches. Now, studies of zebrafish that are mutant for integrin- $\alpha 5$ have revealed a new link between integrins and face patterning.

The mutant animals, which the authors isolated in a screen for cartilage defects, lack the first pharyngeal pouch and have defects in the cartilage structures derived from the second pharyngeal arch - a phenotype that is linked to the loss of Integrin- $\alpha 5$ function specifically in the first pharyngeal pouch. Embryos with cartilage defects also have muscle and nerve defects within the same face region. The fact that transplanted wild-type pouch endoderm, but not crest, can rescue the cartilage defects of a mutant shows that the gene is required in the pouch rather than in the cartilage. So, it is likely that the cartilage, muscle and nerve defects seen in the mutants are secondary to the pouch defects. Moreover, cell-fate mapping showed that cells closer to the first pouch are the most affected by the mutation and that poor compaction and abnormal death of the neural crest cells that surround the pouch probably cause the cartilage defects.

The most striking feature of the integrin- $\alpha 5$ mutant phenotype is the hierarchical way in which it triggers face patterning: it seems that the Integrin- $\alpha 5$-dependent endodermal pouch patterns the crest, which in turn might control the development of neighbouring muscles and nerves. Could this interconnected developmental mechanism have shaped the evolution of the head, by allowing the tissues of each head section to vary in size or shape in a coordinated fashion? Perhaps, although it remains to be shown that similar hierarchical relationships hold true for other parts of the vertebrate head.

Tanita Casci

\section{6) References and links}

ORIGINAL RESEARCH PAPER Crump, J. G. et al. An integrin-dependent role of pouch endoderm in hyoid cartilage development. PLoS Biol. 2, E244 (2004)

WEB SITE

Charles Kimmel's laboratory:

http://www.neuro.uoregon.edu/ionmain/htdocs/ faculty/kimmel.html

\section{A better bodyclock}

A new approach that uses gene-expression profiles to measure individual body time (BT) promises to make a big difference to the way we will administer drugs in the future.

Having access to an accurate clock or watch is pretty important - as anybody who has been late for an exam or an interview because of an unreliable timepiece can confirm! It can also be important to read the correct time from the body's circadian clock: administering drugs at an inappropriate BT can cause side-effects, whereas at the correct BT a drug's potency might be maximized and its toxicity minimized.

To address the lack of clinically applicable methods of BT detection, Hiroki Ueda and colleagues aimed to create a standard 'molecular timetable' of gene-expression profiles. First, they monitored genome-wide expression profiles from pooled liver samples, taken from BALB/c mice over a 2-day period, under either 12-hour cycles of light and dark, or constant dark conditions. The peak expression times for the 168 genes that had high circadian rhythmicity were distributed over a 24-hour period and did not differ between the two light/dark regimes, which indicated that they could provide a direct measure of the endogenous state of the circadian clock.

Next, the authors used a statistical approach that allowed the BT information to be extracted from the expression profiles of the time-indicating genes. The gradually changing expression profiles of night-indicating and day-indicating genes can be accurately modelled by the best-fitted cosine curve.

Their verification in independent samples indicated that this method could provide accurate estimates of BT. But how clinically useful is such a timetable? In mice, at least, the molecular timetable easily detected rhythm disorders in four $\mathrm{Clock}^{-/}$mutants compared with wild-type mice. Moreover, given that circadian rhythmicity could also be detected in $\mathrm{C} 3 \mathrm{H}$ mice, with the timetable that was calibrated in $\mathrm{BALB} / \mathrm{c}$ mice, it seems this method is robust to changes in genetic background.

So, Ueda and colleagues might have provided a robust means of estimating BT from a single-point measurement: a long-held ambition for many clinicians. Of course, it is a long way from these experiments in inbred mouse strains to a routine clinical tool for humans, but given the number of genes in humans that show circadian expression patterns, there is certainly no obvious reason why the same approach would not be applicable.

\section{Nick Campbell}

\section{6) References and links}

ORIGINAL RESEARCH PAPER Ueda, H. R. et al. Molecular-timetable methods for detection of body time and rhythm disorders from single-time-point genome-wide expression profiles. Proc. Natl Acad. Sci. USA 101, 11227-11232 (2004) WEB SITE

Hiroki Ueda's laboratory: http://www.riken.go.jp/engn/r-world/research/lab/cdb/ system/Online links

(Printed image adapted from one provided by Hiroki Ueda.) 